\title{
Some Properties of Fuzzy AB-ideal of AB-algebras
}

\author{
Areej Tawfeeq Hameed \\ Baneen Najah Abaas \\ Department of Mathematics, Faculty of Education for Girls, University of Kufa. \\ areej238@gmail.com \\ areejtawfeeq@uokufa.edu.iq \\ Recived : $8 \backslash 10 \backslash 2017$ \\ Revised : $29 \backslash 10 \backslash 2017$ \\ Accepted : $1 \backslash 1112017$
}

Available online : $20 / \mathbf{1} / 2018$

DOI: 10.29304/jqcm.2018.10.1.333

\begin{abstract}
:
In this research we have introduced the concept of fuzzy $\mathrm{AB}$-ideal of AB-algebra and also we proved some relevant characteristics and theories. We also studied the fuzzy relations on AB-algebras and fuzzy derivations AB-ideals. We presented the characteristics and theories that illustrat the two concepts that is promted us to study Cartesian Product of fuzzy derivations AB-ideals.
\end{abstract}

\section{Keywords:}

Fuzzy derivation AB-ideal of AB-algebras, Cartesian Product of Fuzzy Derivations AB-ideals .

Mathematics Subject Classification: 06F35, 03G25, 03B52.

\section{Introduction:}

The notion of BCC-algebras was proposed by W.A. Dudek in ([9],[10],[11]). S.S. Ahn and H.S. Kim have introduced the notion of QSalgebras. A.T. Hameed and et al ([7],[8]) introduced the notions of QS-ideal and fuzzy QS-ideal of QS-algebra. A.T. Hameed, and et al have introduced the notion of fuzzy QSideal of QS-algebra in [1]. A.T. Hameed introduce new of abstract algebras: is called KUS-algebras and define its ideals as KUSalgebras in ([5]). In 2017, A.T. Hameed and B.N. Abbas. introduced the notion of ABideals in $\mathrm{AB}$-algebras, described connections between such ideal and congruences and some properties of AB-algebra in ([2],[3]). A.T. Hameed and B.N. Abbas, considered the fuzzification of AB-ideals in

$\mathrm{AB}$-algebras [4]. In this paper, we introduce the notion of fuzzy derivation on $\mathrm{AB}$ algebras and obtain some of related properties and we characterized Cartesian Product of Fuzzy Derivations AB-ideals .

\section{Preliminaries:}

We review some definitions and properties that will be useful in our results. 
Definition 1.1.([2],[3]) Let $\mathrm{X}$ be a set with a binary operation " $*$ " and a constant 0 .Then $(\mathrm{X}, *, 0)$ is called an AB-algebra if the following axioms satisfied: for all $\mathrm{x}, \mathrm{y}, \mathrm{z}$ $\in X$ :

(i) $((x * y) *(z * y)) *(x * z)=0$,

(ii) $0 * x=0$,

(iii) $x * 0=x$,

Note that: Define a binary relation $(\leq)$ on $\mathrm{X}$ by $x * y=0$ if and only if, $x \leq y$.

$\operatorname{Then}(\mathrm{X}, \leq)$ is a partially ordered set.

Proposition 1.2.([2],[3]) In any AB-algebra $\mathrm{X}$, for all $\mathrm{x}, \mathrm{y}, \mathrm{z} \in \mathrm{X}$, the following properties hold:

(1) $(x * y) * x=0$.

(2) $x \leq y$ implies $x * z \leq y * z$.

(3) $x \leq y$ implies $z * y \leq z * x$.

Remark 1.3.([2],[3]) An AB-algebra is satisfies for all $\mathrm{x}, \mathrm{y}, \mathrm{z} \in \mathrm{X}$

(1) $(x * y) * z=(x * z) * y$

(2) $(x *(x * y)) * y=0$.

Definition 1.4. ([2],[3]) Let $\mathrm{X}$ be an ABalgebra and $\mathrm{I} \subseteq \mathrm{X}$. I is called an AB-ideal of $\mathbf{X}$ if it satisfies the following conditions:

(i) $0 \in \mathrm{I}$,

(ii) $(x * y) * z \in \mathrm{I}$ and $y \in \mathrm{I}$ imply $x * z \in \mathrm{I}$

Definition 1.5.([2],[3]) For an AB-algebra X, we denote $x \wedge y=y *(y * x)$, for all $\mathrm{x}, \mathrm{y}$ $\in \mathrm{X}$,

$x \wedge y \leq x, y$.

Definition 1.6.([2],[3]) An AB-algebra is said to be commutative if and only if, satisfies for all $\mathrm{x}, \mathrm{y} \in \mathrm{X}, x *(x * y)=$ $y *(y * x)$, i.e, $x \wedge y=y \wedge x$.
Definition 1.7.([2],[3]) Let $X$ be an AB-algebra. A mapping $\mathrm{d}: \mathrm{X} \rightarrow \mathrm{X}$ is a left-right derivation (briefly, $(1, r)$-derivation) of $\mathrm{X}$,

if it satisfies the identity

$d(x * y)=(d(x) * y) \wedge(x * d(y))$, for all $\mathrm{x}, \mathrm{y} \in \mathrm{X}$,

if $\mathrm{d}$ satisfies the identity $d(x * y)=$

$$
(x * d(y)) \wedge(d(x) * y) \text {, for all } \mathrm{x}, \mathrm{y} \in
$$

$\mathrm{X}$

Then $\mathrm{d}$ is a right-left derivation (briefly,

$(\mathrm{r}, \mathrm{l})$-derivation) of $\mathrm{X}$.

Moreover, if $\mathrm{d}$ is both a $(1, \mathrm{r})$ and

$(r, 1)$-derivation, then $\mathrm{d}$ is a derivation of $\mathrm{X}$.

Example 1.8. Let $\mathrm{X}=\{0,1,2,3\}$ be an $\mathrm{AB}$-algebra in which the operation $(*)$ is defined as follows:

\begin{tabular}{|l|l|l|l|l|}
\hline$*$ & $\mathbf{0}$ & $\mathbf{1}$ & $\mathbf{2}$ & $\mathbf{3}$ \\
\hline $\mathbf{0}$ & $\mathbf{0}$ & $\mathbf{0}$ & $\mathbf{0}$ & $\mathbf{0}$ \\
\hline $\mathbf{1}$ & $\mathbf{1}$ & $\mathbf{0}$ & $\mathbf{1}$ & $\mathbf{0}$ \\
\hline $\mathbf{2}$ & $\mathbf{2}$ & $\mathbf{2}$ & $\mathbf{0}$ & $\mathbf{0}$ \\
\hline $\mathbf{3}$ & $\mathbf{3}$ & $\mathbf{3}$ & $\mathbf{1}$ & $\mathbf{0}$ \\
\hline
\end{tabular}

Define a map $\mathrm{d}: \mathrm{G} \rightarrow \mathrm{G}$ by

$$
d(x)=\left\{\begin{array}{l}
0 \text { if } x=0,1,3 \\
2 \text { if } x=2 .
\end{array}\right.
$$

And define a map d $\mathrm{d}^{*} \mathrm{G} \rightarrow \mathrm{G}$

$\operatorname{byd}^{*}(x) \begin{cases}0 & \text { if } x=0,1 \\ 2 & \text { if } x=2,3\end{cases}$

Then it is easily checked that $\mathrm{d}$ is both a $(1, \mathrm{r})$ and $(r, 1)$-derivation of $\mathrm{G}$ and $\mathrm{d}^{*}$ is $\mathrm{a}(\mathrm{r}, \mathrm{l})$ derivation but not a (1,r)-derivation of $\mathrm{G}$.

Definition 1.9.([2],[3]) A derivation of an $A B$-algebra is said to be regular if $\mathrm{d}(0)=0$.

Definition 1.10.([4]) A fuzzy subset $\mu$ of $\mathrm{AB}$-algebra $\mathrm{X}$ is called a fuzzy ABsubalgebra of $X$ if $\mu(x * y) \geq \min \{\mu(x), \mu(y)\}$, for all $x, y \in$ $\mathrm{X}$. 
Definition 1.11.([4]) A fuzzy subset $\mu$ of AB-algebra $X$ is called a fuzzy AB-ideal of X if it satisfies :

$\left.\mathrm{FAB}_{1}\right) \mu(0) \geq \mu(\mathrm{x})$

$\left.\mathrm{FAB}_{2}\right) \mu(\mathrm{x} * \mathrm{z}) \geq \min \{\mu(\mathrm{x} *(\mathrm{y} * \mathrm{z})), \mu(\mathrm{y})\}$, for all $\mathrm{x}, \mathrm{y}, \mathrm{z} \in \mathrm{X}$.

Definition 1.12. [6]. A fuzzy $\mu$ is called a fuzzy relation on any set $S$, if $\mu$ is a fuzzy subset $\mu: \mathrm{X} \times \mathrm{X} \rightarrow[0,1]$.

Definition 1.13. [6].If $\mu$ is a fuzzy relation on a set $S$ and is a fuzzy subset of $X$, then $\mu$ is a fuzzy relation on $\beta$ if $\mu\{\mathrm{x}, \mathrm{y}\} \leq \min$ $(\beta(\mathrm{x}), \beta(\mathrm{y})), \forall \mathrm{x}, \mathrm{y} \in \mathrm{X}$.

Definition 1.14 [6].Let $\mu$ and $\beta$ be a fuzzy subset of a set $\mathrm{X}$, the Cartesian product of $\mu$ and $\beta$ is defined by $(\mu \times \beta)(\mathrm{x}, \mathrm{y})=$ $\min \{\mu(\mathrm{x}), \beta(\mathrm{y})\}, \quad \forall \mathrm{x}, \mathrm{y} \in \mathrm{X}$.

Lemma 1.15 [6]. Let $\mu$ and $\beta$ be a fuzzy subset of a set $X$, then

(i) $\mu \times \beta$ a fuzzy relation on $\mathrm{X}$,

(ii) $(\mu \times \beta)_{t}=\mu_{t} \times \beta_{t}, \forall \mathrm{t} \in[0,1]$.

\section{2.(Fuzzy) Derivations AB-Ideals on AB-Algebras:}

We review the definition of fuzzy derivations AB-algebra and study some properties of it.

Definition 2.1. Let $(X, *, 0)$ be an AB-algebra. and $d: X \rightarrow X$ be a self map. A non-empty subset $\mathrm{A}$ of an $\mathrm{AB}$-algebra $X$ is called left derivations AB-ideal of $X$, If it satisfies the following conditions:

1) $0 \in A$,

2) $d(x) *(y * z) \in \mathrm{A}$, and $d(y) \in \mathrm{A}$ implies $d(x * z) \in \mathrm{A}$.
Definition2.2. Let $(X, *, 0)$ be an AB-algebra. and $d: X \rightarrow X$ be a self map. A non-empty subset $\mathrm{A}$ of an $\mathrm{AB}$ - algebra $X$ is called right derivations AB-ideal of $\mathbf{X}$, If it satisfies the following conditions:

1) $0 \in \mathrm{A}$,

2) $x * d(y * z) \in \mathrm{A}$, and $d(y) \in \mathrm{A}$ implies $d(x * z) \in \mathrm{A}$.

Definition 2.3. Let $(\mathrm{X} ; *, 0)$ be an $\mathrm{AB}$-algebra. and $d: X \rightarrow X$ be a self map. A non-empty subset $\mathrm{A}$ of an AB-algebra $X$ is called derivations $\quad A B$-ideal of If it satisfies the following conditions:

1) $0 \in \mathrm{A}$,

2) $d((x * y) * z) \in \mathrm{A}$, and $d(y) \in \mathrm{A}$

implies $d(x * z) \in \mathrm{A}$.

Definition 2.4. Let $(X, *, 0)$ be an AB-algebra. and $d: X \rightarrow X$ be a self map. A non-empty subset $\mathrm{A}$ of an AB-algebra $X$ is called a fuzzy derivations $\mathbf{A B}$-ideal of $X$ If it satisfies the following conditions:

1) $\mu(0) \geq \mu(x)$;

2) $\mu(d(x * z)) \geq \min \{\mu(d(x *(y * z)), \mu$ $(\mathrm{d}(\mathrm{y}))\}^{\prime \prime}$, for all $\mathrm{x}, \mathrm{y}, \mathrm{z} \in \mathrm{X}$

Example 2.5. Let $\mathrm{X}=\{0,1,2,3\}$ be an $\mathrm{AB}$ algebra in which the operation (*) is defined as follows:

\begin{tabular}{|c|c|c|c|c|}
\hline$*$ & $\mathbf{0}$ & $\mathbf{1}$ & $\mathbf{2}$ & $\mathbf{3}$ \\
\hline $\mathbf{0}$ & $\mathbf{0}$ & $\mathbf{0}$ & $\mathbf{0}$ & $\mathbf{0}$ \\
\hline $\mathbf{1}$ & $\mathbf{1}$ & $\mathbf{0}$ & $\mathbf{1}$ & $\mathbf{0}$ \\
\hline $\mathbf{2}$ & $\mathbf{2}$ & $\mathbf{2}$ & $\mathbf{0}$ & $\mathbf{0}$ \\
\hline $\mathbf{3}$ & $\mathbf{3}$ & $\mathbf{3}$ & $\mathbf{1}$ & $\mathbf{0}$ \\
\hline
\end{tabular}

Define a map $\mathrm{d}: \mathrm{X} \rightarrow \mathrm{X}$ by

$$
d(x)=\left\{\begin{array}{l}
0 \text { if } x=0,1,3, \\
2 \text { if } x=2 .
\end{array}\right.
$$

Define a fuzzy set $\mu: X \rightarrow[0,1]$ by $\mu(\mathrm{d}$ $(0))=t_{0}, \mu(d(1))=t_{1}, \mu\left(d(2)=\mu(d(3))=t_{2}\right.$ Where $t_{0}, t_{1}, t_{2} \in[0,1]$, with $t_{0}>t_{1}>t_{2}$. Routine calculations give that $\mu$ is not fuzzy left (right)derivations $\mathrm{AB}$-ideal of $\mathrm{AB}$-algebra. 
Example 2.6.Consider the set $\mathrm{X}=\{0,1,2,3,4,5\}$ with the operation defined by the following table:

\begin{tabular}{|l|l|l|l|l|l|l|}
\hline$*$ & $\mathbf{0}$ & $\mathbf{1}$ & $\mathbf{2}$ & $\mathbf{3}$ & $\mathbf{4}$ & $\mathbf{5}$ \\
\hline $\mathbf{0}$ & $\mathbf{0}$ & $\mathbf{0}$ & $\mathbf{0}$ & $\mathbf{0}$ & $\mathbf{0}$ & $\mathbf{0}$ \\
\hline $\mathbf{1}$ & $\mathbf{1}$ & $\mathbf{0}$ & $\mathbf{0}$ & $\mathbf{0}$ & $\mathbf{0}$ & $\mathbf{1}$ \\
\hline $\mathbf{2}$ & $\mathbf{2}$ & $\mathbf{2}$ & $\mathbf{0}$ & $\mathbf{0}$ & $\mathbf{1}$ & $\mathbf{1}$ \\
\hline $\mathbf{3}$ & $\mathbf{3}$ & $\mathbf{2}$ & $\mathbf{1}$ & $\mathbf{0}$ & $\mathbf{1}$ & $\mathbf{1}$ \\
\hline 4 & 4 & 4 & 4 & 4 & $\mathbf{0}$ & $\mathbf{1}$ \\
\hline 5 & 5 & 5 & 5 & 5 & 5 & 0 \\
\hline
\end{tabular}

define $\mathrm{d}(\mathrm{x}): \mathrm{X} \rightarrow \mathrm{X}$ byd $(x)=\left\{\begin{array}{cc}0 & \text { if } \mathrm{x}=0,1,2,3,4 \\ 5 & \text { if } \mathrm{x}=5\end{array}\right.$ define a fuzzy set $\mu: X \rightarrow[0,1]$ by $\mu(d(0))=t_{0}, \mu(d$ (1) $)$ - thlgebra $X$ is also fuzzy left derivations $\mu(d(1))=\mu(d(2))=t_{2}, \mu(d(3))=\mu(d(4))=t_{3}$. Where $t_{0}, t_{1}, t_{2}, t_{3} \in[0,1]$, with $t_{0}>t_{1}>t_{2}>t_{3}$. Routipe calculations give that $\mu$ is a fuzzy derivations $\mathrm{AB}$-ideal ofAB-ideals of AB-algebra $\mathrm{X}$, then AB-algebra.

Theorem 2.7. Let $\mu$ be a fuzzy derivations AB-ideal of AB-algebra $X$.

(1) If $x \leq d(y)$, then $\mu(d(x)) \geq \mu(d(y))$,

(2) If $x * y \leq d(y)$, then $\mu(d(x * y)) \geq \mu(d(y))$,

(3) If $(x * y) *(z * y) \leq d(x * z)$, then $\mu(\mathrm{d}$ $((x * y) *(z * y))) \geq \mu(d(z * x))$,

(4) If $\mu(d(x * y))=\mu(d(0))$, then $\mu(d(x)) \geq \mu(d(y))$

\section{Proof :}

(1) Let $x \leq d(y)$, and since $d(y) \leq y$, hence $x \leq y$, i.e. $x * y=0$, then

$$
\begin{aligned}
& \mu(d(x))=\mu(d(x * 0)) \\
& \geq \min \{\mu(d(x * y) * 0), \mu(d(y))\}
\end{aligned}
$$

(from Definition $(2.4(2))$ )

$$
\begin{aligned}
& =\min \{\mu(d(x * y)), \mu(d(y))\} \\
& =\min \{\mu(d(0), \mu(d(y))\}=\mu(d(y))
\end{aligned}
$$
AB-ideal. Proof:

Let $\left\{\mu_{i}\right\}$ be a family of fuzzy derivations

(2) Let $x * y \leq d(y)$, Then by Theorem (2.7(1)) we get, $\mu(d(x * y)) \geq \mu(d(x))$ (3) Let $(x * y) *(z * y) \leq d(x * z)$, by (Theorem $(2.7(1))$ we get, $\mu(d((x * y) *(z * y))) \geq \mu(d(z * x))$

(4) Let $\mu(d(x * y))=\mu(d(0))$, then $\mu(d(x))=\mu(d(x * 0)) \geq \min \{\mu(d(x *$ $y) * 0), \mu(d(y))\}=\min \{\mu(d(x *$ $y)), \mu(d(y))\}$ $=\min \{\mu(0), \mu(d(y))\}=\mu(d(y))$.

Proposition 2.8. The intersection of any set of fuzzy derivations $\mathrm{AB}$-ideals of $\forall \mathrm{x}, \mathrm{y}, \mathrm{z} \in \mathrm{X}\left(\cap \mu_{i}\right)(0)=\inf \left(\cap \mu_{i}(0)\right)$ $\geq \inf \left(\mu_{i}(d(x))=\left(\bigcap \mu_{i}\right)(d(x))\right.$ and $\left(\bigcap \mu_{i}\right)(d(x * z))=\inf \left(\mu_{i}(d(x * z))\right.$ $\geq \inf \left(\min \left\{\mu_{i}(d(x * y)\right.\right.$

$\left.* z)), \mu_{i}(d(y))\right\}$

$=\min \left\{\inf \left(\mu_{i}(d(x * y) * z)\right), \mu_{i}(d(y))\right\}$

$$
=\min \left\{\left(\cap \mu_{i}\right)(d(x * y) *\right.
$$

$\left.z),\left(\cap \mu_{i}\right)(d(y))\right\}$.

Theorem 2.9. Let $\mu$ be a fuzzy set in $X$, then $\mu$ is a fuzzy derivations $\mathrm{AB}$-ideal of $\mathrm{X}$ if and only if it satisfies : $\forall \alpha \in[0,1]), U(\mu, \alpha) \neq \varphi$ implies $U(\mu, \alpha)$ is $A B$-ideal of $X$, where $\mathrm{U}(\mu, \alpha)=\{\mathrm{x} \in \mathrm{X} / \mu(d(x)) \geq \alpha\}$.

Proof: Assume that $\mu$ is a fuzzy derivations AB-ideal of $X$, let $\alpha \in 0,1$ be such that $\mathrm{U}(\mu, \alpha) \neq \varphi$ and $\mathrm{x}, \mathrm{y} \in \mathrm{X}$, such that $\mathrm{x} \in \mathrm{U}(\mu, \alpha)$ then $\mu(d(x)) \geq \alpha$, and so by $\left(f l_{2}\right) \quad \mu(d(0))=\mu(d(0 * y)) \geq$ $\min \{\mu(d(0 * x) * y), \quad \mu(d(x)) \quad=$ $\min \{\mu(d(0) * y), \mu(d(x))$

$=\min \{\mu(0 * y), \mu(d(x))=\min \{\mu(0)$, $\mu(d(x))=\alpha$, hence $0 \in \mathrm{U}(\mu, \alpha)$.

Let $d(x *(y * z)) \in \mathrm{U}(\mu, \alpha)$ and $d(y) \in$ $\mathrm{U}(\mu, \quad \alpha), \quad$ by $\left(F L_{2}\right) \quad \mu(d(x * z)) \geq$ $\min \{\mu(d(x * y) * z), \mu(d(y))\}=\alpha$, so that $\mathrm{x}^{*} \mathrm{z} \in \mathrm{U}(\mu, \alpha)$. 
Hence $U(\mu, \alpha)$ is AB-ideal of $X$.

Conversely, Let $U(\mu, \alpha)$ is $A B$-ideal of $X$, let $\mathrm{x}, \mathrm{y}, \mathrm{z} \in \mathrm{X}$ be such that $\mu(\mathrm{d}(\mathrm{x} * \mathrm{z}))>\min \{\mu(\mathrm{d}(\mathrm{x} * \mathrm{y}) * \mathrm{z}), \mu(\mathrm{d}(\mathrm{y}))\}$, $\operatorname{taking} \beta_{0}=\frac{1}{2}\{\mu(d(x * z))+\min \{\mu(d(x *$ $y) * z), \mu(d(y))\}\}$, we have $\beta_{0} \in[0,1]$, $\mu(d(x * z))$

$\left.<\beta_{0}<\min \{\mu(d(x * y) * z), \mu(d(y))\}\right\}$, it follows that $d(x * y) * z \in \mathrm{U}\left(\mu, \beta_{0}\right)$ and $\mathrm{y} \in \mathrm{U}\left(\mu, \beta_{0}\right)$, this this is a contradiction and therefore $\mu$ is a fuzzy derivations AB-ideal of $\mathrm{X}$.

\section{Cartesian Product of Fuzzy Derivations AB-ideals:}

The following definitions introduce the notion of Cartesian Product of Fuzzy Derivations AB-ideals and some Properties of it.

Definition 3.1. Let $\mu$ and $\beta$ be a fuzzy derivations subset of a set X,the Cartesian product of $\boldsymbol{\mu}$ and $\boldsymbol{\beta}$ is defined by $(\mu \times \beta)(d(x, y))$

$=\min \{\mu(d(x)), \beta(d(y))\}, \forall \mathrm{x}, \mathrm{y} \in \mathrm{X}$.

Definition 3.2. If $\beta$ is a fuzzy derivations subset of a set $\mathrm{X}$, the strongest fuzzy relation on $\mathrm{X}$, that is a fuzzy derivations relation on $\boldsymbol{\beta}$ is $\boldsymbol{\mu}_{\boldsymbol{\beta}}$ given by $\mu_{\beta}(d(x, y))=\min \{\beta(d(x)), \beta(d(y))\}$, $\forall \mathrm{x}, \mathrm{y} \in \mathrm{X}$.

Proposition 3.3. For a given fuzzy derivations subset $\beta$ of AB-algebra $\mathrm{X}$, let $\mu_{\beta}$ be the strongest fuzzy derivations relation on $\mathrm{X}$. If $\mu_{\beta}$ is a fuzzy derivations $\mathrm{AB}$-ideal of $\mathrm{X} \times \mathrm{X}$ then $\beta(d(x)) \leq \beta(d(0))=\beta(0), \forall \mathrm{x} \in \mathrm{X}$.

Proof:

Since $\mu_{\beta}$ is a fuzzy derivation $\mathrm{AB}$-ideal of $\mathrm{X}$ $\times \mathrm{X}$ it follows from $\left(\mathrm{F}_{1}\right)$ that

$\mu_{\beta}(\mathrm{x}, \mathrm{x})=\min \{\beta(d(x)), \beta(d(x))\} \leq \beta$

$(d(0,0))$

$=\min \{\beta(d(0), \beta(d(0)\}$ where $(0,0) \in \mathrm{X} \times \mathrm{X}$ then $\beta(d(x)) \leq \beta(d(0))=\beta(0)$.
Remark 3.4. Let $\mathrm{X}$ and $\mathrm{Y}$ be $\mathrm{AB}$-algebras, we define $*$ on $\mathrm{X} \times \mathrm{Y}$ by

$(x, y) *(u, v)(x * u, y * v), \forall(x, y),(u, v)$

$\in \mathrm{X} \times \mathrm{Y}$ then clearly $\left(\mathrm{X} \times \mathrm{Y},{ }^{*},(0,0)\right)$ is an

AB-algebra.

Theorem 3.5. Let $\mu$ and $\beta$ be a fuzzy derivations $\mathrm{AB}$-ideals of $\mathrm{AB}$-algebra $\mathrm{X}$, then $\mu$ $\times \beta$ is a fuzzy derivations $A B$-ideal of $X \times X$.

Proof:

(1) $(\mu \times \beta)(\mathrm{d}(0,0))=\min \{\mu(0), \beta(0)$

$\geq \min \{\mu(d(\mathrm{x})), \beta(d(\mathrm{x}))\}=\mu \times \beta(\mathrm{d}(\mathrm{x}, \mathrm{y}))$

$\forall \mathrm{x}, \mathrm{y} \in \mathrm{X} \times \mathrm{X}$.

(2) Let $\left(x_{1}, x_{2}\right),\left(y_{1}, y_{2}\right),\left(z_{1}, z_{2}\right) \in \mathrm{X} \times \mathrm{X}$, then

$\mu \times \beta\left(x_{1} * z_{1}, x_{2} * z_{2}\right)=\min \left\{\mu\left(\mathrm{d}\left(x_{1}, z_{1}\right) *\right.\right.$

$\beta\left(\mathrm{d}\left(x_{2}, z_{2}\right)\right)$

$\geq \min \left\{\mu\left(d\left(x_{1} *\left(y_{1} * z_{1}\right)\right), \mu\left(d\left(y_{1}\right)\right)\right.\right.$,

$\beta\left(d\left(x_{2} *\left(y_{2} * z_{2}\right)\right), \beta\left(d\left(y_{2}\right)\right)\right\}$

$=\min \left\{\min \left\{\mu\left(d\left(x_{1} *\left(y_{1} * z_{1}\right)\right)\right.\right.\right.$,

$\beta\left(d\left(x_{2} *\left(y_{2} *\right.\right.\right.$

$\left.\left.\left.z_{2}\right)\right)\right\}, \min \left\{\mu\left(d\left(y_{1}\right)\right), \beta\left(d\left(y_{2}\right)\right)\right\}$

$=\min \left\{\mu \times \beta\left(\left(d\left(x_{1} *\left(y_{1} * z_{1}\right)\right)\right.\right.\right.$,

$\left.\left.\left(d\left(x_{2} *\left(y_{2} * z_{2}\right)\right)\right), \mu \times \beta\left(d\left(y_{1}\right)\right),\left(d\left(y_{2}\right)\right)\right)\right\}$

Hence $\mu \times \beta$ is a fuzzy derivations AB-ideal of $\mathrm{X} \times \mathrm{X}$.

Theorem 3.6. Let $\beta$ be a fuzzy derivations subset of $A B$-algebra $X$ and let $\mu_{\beta}$ be the strongest fuzzy derivations relation on then $\beta$ is a fuzzy derivations $\mathrm{AB}$-ideal of $\mathrm{X}$ if and only if $\mu_{\beta}$ is a fuzzy derivations $A B$-ideal of $X \times X$.

Proof:

Let $\beta$ be a fuzzy derivations AB-ideal of $X$.

(1) From $\left(F_{1}\right)$, we get $\mu_{\beta}(0,0)$

$=\min \{\beta(d(0)), \beta(d(0))\}=\min \{\beta(0), \beta(0)\}$

$\left.\geq \min \{\beta(d(\mathrm{x})), \beta(d(\mathrm{y}))\}=\mu_{\beta}(d(\mathrm{x})), d(\mathrm{y})\right)$,

$\forall \mathrm{x}, \mathrm{y} \in \mathrm{X} \times \mathrm{X}$.

(2) $\forall\left(x_{1}, x_{2}\right),\left(y_{1}, y_{2}\right),\left(z_{1}, z_{2}\right) \in \mathrm{X} \times \mathrm{X}$, we have from $\left(F_{2}\right)$

$\mu_{\beta}\left(x_{1} * z_{1}, x_{2} * z_{2}\right)=\min \left\{\beta\left(\mathrm{d}\left(x_{1} *\right.\right.\right.$

$\left.\left.z_{1}\right), \beta\left(\mathrm{d}\left(x_{2} * z_{2}\right)\right)\right\}$

$\geq \min \left\{\beta\left(d\left(x_{1} *\left(y_{1} * z_{1}\right)\right), \beta\left(d\left(y_{1}\right)\right)\right.\right.$,

$\beta\left(d\left(x_{2} *\left(y_{2} * z_{2}\right)\right), \beta\left(d\left(y_{2}\right)\right)\right\}$

$=\min \left\{\min \left\{\beta\left(d\left(x_{1} *\left(y_{1} * z_{1}\right)\right)\right.\right.\right.$,

$\beta\left(d\left(x_{2} *\left(y_{2} *\right.\right.\right.$

$\left.\left.\left.\left.z_{2}\right)\right)\right\}, \min \left\{\beta\left(d\left(y_{1}\right)\right), \beta\left(d\left(y_{2}\right)\right)\right\}\right\}$

$=\min \left\{\mu_{\beta}\left(\left(d\left(x_{1} *\left(y_{1} * z_{1}\right)\right)\right.\right.\right.$,

$\left.\left.\left(d\left(x_{2} *\left(y_{2} * z_{2}\right)\right)\right), \mu_{\beta}\left(d\left(y_{1}\right)\right),\left(d\left(y_{2}\right)\right)\right)\right\}$ 
Hence $\mu_{\beta}$ is a fuzzy derivations $\mathrm{AB}$-ideal of $\mathrm{X} \times \mathrm{X}$.

Conversely, let $\mu_{\beta}$ be a fuzzy derivations

AB-ideal of $X \times X$,

(1) $\forall(x, y) \in X \times X$, we have,

$\min \{\beta(0), \beta(0)\}=\mu_{\beta}(x, y)=\min \{\beta(\mathrm{x}), \beta(\mathrm{y})\}$,

It follows that $\beta(0) \geq \beta(\mathrm{x}) \forall \mathrm{x} \in \mathrm{X}$,

(2) Let $\left(\mathrm{x}_{1}, \mathrm{x}_{2}\right),\left(\mathrm{y}_{1}, \mathrm{y}_{2}\right),\left(\mathrm{z}_{1}, \mathrm{z}_{2}\right) \in \mathrm{X} \times \mathrm{X}$, then

$\min \left\{\beta\left(\mathrm{d}\left(x_{1} * z_{1}\right), \beta\left(\mathrm{d}\left(x_{2} * z_{2}\right)\right)\right\}\right.$

$=\mu_{\beta}\left(\mathrm{d}\left(x_{1} * z_{1}\right), d\left(x_{2} * z_{2}\right)\right)$

$\geq$

$\min \left\{\mu_{\beta}\left(d\left(\mathrm{x}_{1}, \mathrm{x}_{2}\right) *\left(\left(\mathrm{y}_{1}, \mathrm{y}_{2}\right) *\left(z_{1}, z_{2}\right)\right)\right), \mu_{\beta}(d(\right.$

$\left.\left.\left.y_{1}\right), d\left(y_{2}\right)\right)\right\}$

$=\min \left\{\mu_{\beta}\left(d\left(x_{1} *\left(y_{1} * z_{1}\right)\right), d\left(x_{2} *\left(y_{2} *\right.\right.\right.\right.$

$\left.\left.\left.z_{2}\right)\right), \mu_{\beta}\left(d\left(y_{1}\right), d\left(y_{2}\right)\right)\right\}$

$=\min \left\{\min \left\{\beta\left(d\left(x_{1} *\left(y_{1} * z_{1}\right)\right), \beta\left(d\left(x_{2} *\right.\right.\right.\right.\right.$

$\left.\left.\left.\left(y_{2} * z_{2}\right)\right)\right\}, \min \left\{\beta\left(d\left(y_{1}\right)\right), \beta\left(d\left(y_{2}\right)\right)\right\}\right\}$

$=\min \left\{\beta\left(d\left(x_{1} *\left(y_{1} * z_{1}\right)\right), \beta\left(d\left(y_{1}\right)\right), \beta\left(d\left(x_{2} *\right.\right.\right.\right.$ $\left.\left.\left(y_{2} * z_{2}\right)\right), \beta\left(d\left(y_{2}\right)\right)\right\}$

In particular, if we takex $x_{2}, \mathrm{y}_{2}, z_{2}=0$, then

$\beta\left(\mathrm{d}\left(x_{1} * z_{1}\right) \geq \min \left\{\beta\left(d\left(x_{1} *\left(y_{1} *\right.\right.\right.\right.\right.$

$\left.\left.\left.z_{1}\right)\right), \beta\left(d\left(y_{1}\right)\right)\right\}$.

Hence $\beta$ be a fuzzy derivations AB-ideal of $\mathrm{X}$.

\section{Conclusions and discussion}

1) we presented some properties related to fuzzy AB-ideal of AB- algebra, among these properties, derivations AB-ideals, Cartesian Product of Fuzzy Derivations AB-ideals.

2) We recommend finding other properties for this type of algebra.

\section{References}

[1] A.T. Hameed, A.A. Alfatlawi and A.K. Alkurdi, Fuzzy QS-ideals of QSalgebras, International Journal of Algebra, 11, 1(2017), 43-52.

[2] A.T. Hameed and B.N. Abbas, AB-ideals of AB-algebra, Applied Mathematical Sciences, 11,35(2017), 1715-1723.

[3] A.T. Hameed and B.N. Abbas, Some Properties of AB-ideals of AB-algebra, Algebra Letters, To apper, 2017.

[4] A.T. Hameed and B.N. Abbas, Fuzzy AB-ideals of AB-algebra, To apper, 2017.

[5] A.T. Hameed, Fuzzy ideals of some algebras, $\mathrm{PH}$. Sc. Thesis, Ain Shams University, Faculty of Sciences, Egypt , 2015.

[6] P. Bhattacharye and N.P. Mukheriee, Fuzzy relation and fuzzy group in form, Sci, 36(1985), 267-282.

[7] S.M. Mostafa, A.T. Hameed and A.H. Abed, Fuzzy KUS-ideals of KUSalgebra, Basra, Journal of Science (A), 34, 2 (2016), 73-84.

[8] S.M. Mostafa, M.A. Abdel Naby, F. Abdel-Halim and A.T. Hameed, On KUS-algebra, 7, 3 (2013), 131-144.

[9] W.A. Dudek, A new characterization of ideals in BCC-algebras, Novi Sad J. Math., 29(1999),139-145.

[10] W.A. Dudek, and J. Thomys, On some generalizations of $\mathrm{BCC}$ algebras, Intern. J. Computer Math., 89(2012), 1596-1606.

[11] W.A. Dudek, On BCC-algebras, Logique et Analyse ,129-131(1991), 103111. 


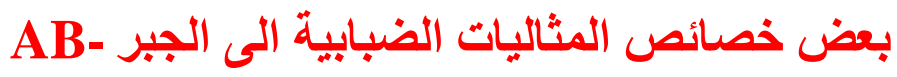

أريج توفيق حميد بنين نجاح عباس

جامعة الكوفة، كلية التربية للبنات، قسم الرياضيات

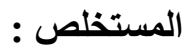

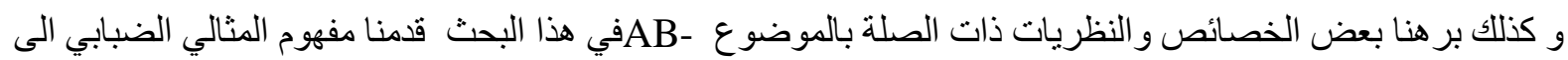

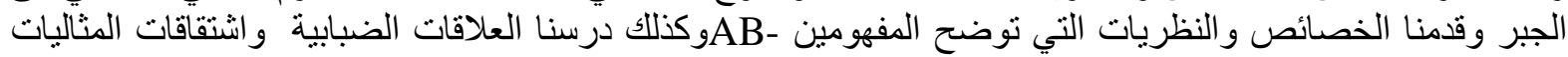
الضبابية الى الجبر -ABما دفعنا الى دراسة الضرب الضئ الديكارتي الى اشتقاقات المثاليات الضبابية الى الجبر 